\title{
Hemmnisse überwiegen
}

\section{Mit dem Ziel Nachhaltigkeit muss sich die Land- und Ernährungswirtschaft stär- ker an den Anforderungen einer umweltgerechten Landnutzung und Wirtschafts- weise, der Sicherung von Einkommen und Arbeitsplätzen sowie einer adäquaten Partizipation orientieren. Die Gestaltungsspielräume sind allerdings von zahlrei- chen wirtschaftlichen und politisch-rechtlichen Rahmenbedingungen abhängig.}

I

Von Guido Nischwitz

n einem dynamischen Spannungsfeld von

Globalisierungs- und Regionalisierungstendenzen verfügen die betroffenen Akteursgruppen über unterschiedliche Steuerungszugänge und Regelungsfähigkeiten. Neben klassischen Akteursgruppen aus Wirtschaft (Agribusiness, Handelsunternehmen, Landwirte) und Politik/ Administration (WTO, EU, Bund, Länder und Kommunen) treten verstärkt gesellschaftspolitische Gruppen (NGOs, Kirchen und Agenda 21-Initiativen) in Erscheinung.

Klassische Regelungsmechanismen von Markt und Hierarchie erscheinen kaum in der Lage, entscheidende Schritte in Richtung einer nachhaltigen Land- und Ernährungswirtschaft einzuleiten. Angesichts des Handlungsdrucks kommt es zur Entfaltung neuer Formen von wirtschaftlichen und sozialen Steuerungs- und Entwicklungsansätzen. Dabei erfährt die lokale und regionale Ebene eine deutliche Aufwertung (vergleiche die Beiträge von Molitor und Witzel/ Tischer in diesem Heft). Die beobachtbare Rückbesinnung auf regionale Wirtschaftskreisläufe, Produktions- und Konsumtionsprozesse wird allerdings mit einer Vielzahl an gegenläufigen ökonomischen Entwicklungsprozessen und hemmenden rechtlichen Rahmenbedingungen konfrontiert.

Die fortschreitende Integration der Nahrungsmittelproduktion in internationale Handelsstrukturen hat eine weitreichende Auflösung gewachsener regionaler Wirtschaftskreisläufe zur Folge. Im Ernährungsgewerbe führt der räumliche und sektorale Konzentrationsprozess in vielen Regionen zu einem Verlust an Weiterverarbeitungsstrukturen (zum Beispiel Schlachtstätten, Molkereien). Zurückzuführen ist dieser Strukturwandel unter anderem auf den anhaltenden Verdrängungswettbewerb. Ein Beispiel ist der ruinöse Preiskampf auf dem deutschen Lebensmittelmarkt, der nach dem Markteintritt des US-Handelsriesen WalMart weiter forciert wird. Darüber hinaus hat die deutsche und europäische Agrarpolitik in den letzten Jahrzehnten bewusst eine sogenannte Strukturbereinigung zu Lasten kleinerer Wirtschaftsstrukturen betrieben.

Sowohl im Ernährungshandwerk als auch im Lebensmittel-Einzelhandel führt der Trend zur Filialisierung und zu Produkten mit hohem Verarbeitungsgrad zur Zentralisierung der Warenbeschaffung und Lieferbeziehungen. Der damit verbundene Rückgang an selbständigen Verarbeitern und Einzelhändlern entzieht der nachhaltigen Regionalisierung mögliche Marktpartner. So beziehen bezeichnenderweise die Öko-Handelsmarken (zum Beispiel Füllhorn des ReweKonzerns) ihre Produkte auf den globalen Märkten (1).

Der verschärfte Wettbewerb auf den Nahrungsmittelmärkten bieten allerdings auch Chancen für eine nachhaltige Regionalisierung von Landund Ernährungswirtschaft. Die wachsenden Anforderungen der Verbraucher an eine durchgängige Qualitätssicherung und Herkunftsgarantie bietet Anknüpfungspunkte für eine regional nachvollziehbare Profilierungsstrategie.

Eine Analyse zentraler politisch-rechtlicher Rahmenbedingungen (2) hat aufgezeigt, dass eine nachhaltige Regionalisierung von Produktion und Vermarktung bislang vor allem hemmenden Einflussgrößen unterliegt. Hier lassen sich unter anderem das deutsche Investitionsförderprogramm ,,Verbesserung der regionalen Wirtschaftsstruktur" oder das EU-Lebensmittelund Hygienerecht anführen.

Fördernde Faktoren sind häufig nur auf Nebeneffekte von Rechtsvorschriften und Fördersysteme zurückzuführen. Die wenigen auf eine Ökologisierung und Regionalisierung ausgerichteten Programme weisen entweder einen begrenzten Regelungsraum oder eine zu geringe finanzielle Ausstattung auf.

Insbesondere mit dem Agenda 2000 Reformpaket hat die EU die Chance für eine umfassende
Neuorientierung der gemeinsamen Agrar- und Strukturpolitik vertan. Es fehlt weiterhin an einer integrierten Gesamtkonzeption, die die traditionellen Zielkonflikte überwindet und langfristige Perspektiven für die Land- und Ernährungswirtschaft eröffnet.

Dennoch gibt es seitens der EU wichtige Impulse für eine nachhaltige Entwicklung des Wirtschaftslebens und von Regionen. Positive Handlungsansätze ergeben sich aus den modifizierten EUStrukturfonds und Gemeinschaftsinitiativen wie LEADER +. Besondere Bedeutung erlangt die Verordnung zur „Förderung des ländlichen Raumes“. Als sogenannte zweite Säule der EU-Agrarpolitik zielt sie auf eine Integration von Agrarstruktur-, Umwelt- und Regionalpolitik. Mit einem jährlichen Finanzvolumen von 4,34 Milliarden Euro macht sie allerdings nur zehn Prozent des gesamten Agrarhaushaltes aus.

Inwieweit diese Ansätze in die Gemeinschaftsaufgabe „Verbesserung der Agrarstruktur“ (GAK) und in die regionalen Entwicklungsprogramme der Bundesländer Eingang finden, bleibt abzuwarten. In der Vergangenheit hat das deutsche Fördersystem wichtige Impulse und Rahmenvorgaben der EU zum Teil nur unzureichend aufgenommen. So zeugt die Neuaufnahme des Fördergrundsatzes „Regionale Verarbeitung und Vermarktung" noch nicht von einer grundlegenden Neuorientierung der GAK.

Es bleibt abzuwarten, ob die partiellen Bemühungen der europäischen und deutschen Politik in Richtung einer nachhaltigen Land- und Ernährungswirtschaft in einer neuen inhaltlichen Positionierung oder in einer Spielwiese für Nachhaltigkeit münden werden

\section{Anmerkungen}

(1) Nischwitz, G./ Molitor, R.: Regionale Nachfragepotentiale in der Produktlinie Ernährung. Schriftenreihe des IÖW 135/98, Berlin 1998.

(2) NABU (Hrsg.): Fördernde und hemmende Faktoren für regionale Produktion und Vermarktung, Bonn 1999 (erstellt vom IÖW Nordrhein-Westfalen).

\section{Der Autor}

Dr. Guido Nischwitz ist Leiter des Forschungsfeldes Regionale Wirtschaftspolitik/Nachhaltige Regionalentwicklung im IÖW.

Kontakt: IÖW Regionalbüro Nordrhein-Westfalen, Völklinger Str. 9, 42285 Wuppertal, Tel. 0202/80530, Fax 0202/83402, E-Mail: guido.nischwitz@wuppertal.ioew.de 
(c) 20I0 Authors; licensee IÖW and oekom verlag. This is an article distributed under the terms of the Creative Commons Attribution Non-Commercial No Derivates License (http://creativecommons.org/licenses/by-nc-nd/3.o/), which permits unrestricted use, distribution, and reproduction in any medium, provided the original work is properly cited. 